\title{
Coded Multi-dimensional Spreading System using the Discrete Fourier Transform
}

\author{
Haesik Kim, Garik Markarian, Member, IEEE, and Valdemar C. da Rocha Jr., Senior Member, IEEE.
}

\begin{abstract}
In this paper a novel coded multi-dimensional spreading technique using the Discrete Fourier Transform (DFT) is introduced. It exploits the DFT characteristic of spreading one symbol in one domain to all the symbols in the corresponding transform domain. Frequency domain channel estimation is performed at the receiver. The performance of a coded twodimensional (2D) DFT spreading communication system is investigated and simulation results are presented, using first a $2 \mathrm{D}$ DFT time-frequency system and then a 2D DFT space-frequency system. The proposed 2D DFT system shows good immunity to narrowband interference and impulse noise and exhibits a significant performance improvement when compared with a coded conventional OFDM system of equivalent size.
\end{abstract}

Index Terms-Discrete Fourier Transform, Coded systems, OFDM, Multi-dimensional spreading.

\section{INTRODUCTION}

$\mathbf{H}$ IGH speed communication systems such as ADSL [1], IEEE 802.16 [2], IEEE 802.11 [3], to mention a few cases, have adopted the discrete Fourier transform (DFT) [4], [5] for multi-carrier transmission. Each point of a DFT acts like a subcarrier in a multi-carrier communication system. In practice, it is much easier to generate subcarriers using the DFT rather than conventional local oscillators. However, there is one more important characteristic of the DFT which is to spread one point to a whole block of points. We notice that although orthogonal frequency division multiplexing (OFDM) systems [6], [7] basically use the DFT for multi-carrier transmission, they also simultaneously spread one symbol to a whole block of points. In this manner OFDM systems naturally provide a diversity gain. The one-dimensional spreading technique is widely used in modern communication systems because it allows multiple-access and offers diversity gain. For example, code division multiple access (CDMA) systems [8] assign specific codes such as a direct sequence code or a frequency hopping code [8] to each mobile station and each base station for sharing a limited resource. In case the spreading technique is used for diversity gain, a distorted symbol can still be recovered from the other non-distorted remaining symbols. The 2-dimensional fast Fourier Transform (2D FFT) technique [4] is not a new technique and is widely used in the area of image signal processing [11]. However its application as a modulation technique is fairly recent and has been investigated in [16], where an uncoded version of it has been shown to performs well under jamming and impulse

Haesik Kim and Garik Markarian are with the Department of Communication Systems, InfoLab21, Lancaster University, Lancaster, UK, LA1 4WA (E-mail: \{H.Kim2,G.Markarian\}@lancaster.ac.uk).

Valdemar C. da Rocha Jr. is with the Department of Electronics and Systems, Federal University of Pernambuco, PO Box 7800, 50711- 970 Recife, PE, Brazil (E-mail: vcr@ufpe.br). noise environments. In [17] an uncoded 2D FFT application for relay communications has been investigated.

In this paper we propose a coded multi-dimensional spreading system using the multi-dimensional DFT and demonstrate its usefulness by means of examples. A coded 2-dimensional time-frequency spreading system, as the first example, is constructed and evaluated following WiMAX specifications and a 2-dimensional space-frequency spreading system is constructed as the second example. As a particular case, the performance of a coded two-dimensional DFT (2D DFT) spreading system is evaluated and compared to that of a conventional 1-dimensional spreading system based on OFDM. In Section II the multi-dimensional DFT is reviewed and its spreading properties are illustrated for the 2-dimensional case. In Section III we propose a coded two-dimensional time-frequency spreading [9] and in Section IV we propose and discuss a coded 2-dimensional space-frequency spreading system. It Section V computer simulation results are presented and the paper ends in Section VI with closing remarks.

\section{The Multi-Dimensional Discrete Fourier TRANSFORM}

The conventional 1-dimensional DFT, which is described by a single variable, admits a natural generalisation in more dimensions [10]. The multi-dimensional DFT has an associated representation by a multi-dimensional array such that for a $d$ dimensional DFT, one element of the corresponding array is denoted as $x_{n_{1}, n_{2}, \ldots, n_{d}}$, with discrete variables $n_{1}, n_{2}, \ldots, n_{d}$, $0 \leq n_{\ell} \leq N_{\ell}-1$, where $N_{\ell}$ denotes a positive integer and $1 \leq \ell \leq d$. The multidimensional DFT [10] having $d$ dimensions is defined as follows.

$$
\begin{aligned}
X_{k_{1}, k_{2}, \ldots, k_{d}}= & \sum_{n_{1}=0}^{N_{1}-1}\left(\omega _ { 1 } ^ { k _ { 1 } n _ { 1 } } \sum _ { n _ { 2 } = 0 } ^ { N _ { 2 } - 1 } \left(\omega_{2}^{k_{2} n_{2}} \ldots\right.\right. \\
& \left.\left.\ldots \sum_{n_{d}=0}^{N_{d}-1} \omega_{d}^{k_{d} n_{d}} x_{n_{1}, n_{2}, \ldots, n_{d}}\right) \ldots\right),
\end{aligned}
$$

where $\omega_{\ell}=e^{-\frac{2 \pi i}{N_{\ell}}}, 0 \leq k_{\ell} \leq N_{\ell}-1,1 \leq \ell \leq d$.

It follows from Equation (1) that each element $x_{n_{1}, n_{2}, \ldots, n_{d}}$ is transformed by the factor $\omega_{\ell}^{k_{\ell} n_{\ell}}, 1 \leq \ell \leq d$, and after the appropriate summations are performed it produces the transformed multidimensional array with elements denoted as $X_{k_{1}, k_{2}, \ldots, k_{d}}$ in a different domain. Similar to the 1-dimensional DFT case, the multi-dimensional inverse DFT having $d$ dimensions is defined as follows [10]. 


$$
\begin{aligned}
x_{n_{1}, n_{2}, \ldots, n_{d}}= & \frac{1}{\prod_{\ell=1}^{d} N_{\ell}} \sum_{k_{1}=0}^{N_{1}-1}\left(\omega _ { 1 } ^ { - k _ { 1 } n _ { 1 } } \sum _ { k _ { 2 } = 0 } ^ { N _ { 2 } - 1 } \left(\omega_{2}^{-k_{2} n_{2}} \ldots\right.\right. \\
& \left.\left.\ldots \sum_{k_{d}=0}^{-N_{d}-1} \omega_{d}^{-k_{d} n_{d}} X_{k_{1}, k_{2}, \ldots, k_{d}}\right) \ldots\right)
\end{aligned}
$$

The calculation process in (2) is analogous to that for calculating the multidimensional DFT in (1). There is however a more compact and elegant manner to represent the multidimensional DFT using vectors.

Let $\mathbf{n}=\left(n_{1}, n_{2}, \ldots, n_{d}\right)$ and let $\mathbf{k}=\left(k_{1}, k_{2}, \ldots, k_{d}\right)$ denote $d$-dimensional vectors, $\mathbf{0} \leq \mathbf{n} \leq \mathbf{N}-1$, where $\mathbf{0} \triangleq(0,0, \ldots, 0), \mathbf{N}-1 \triangleq\left(N_{1}-1, N_{2}-1, \ldots, N_{d}-1\right)$. When written in vectorial notation, Equation (1) is, equivalently, expressed as follows.

$$
X_{\mathbf{k}}=\sum_{\mathbf{n}=0}^{\mathbf{N}-1} e^{-2 \pi i \mathbf{k} \cdot(\mathbf{n} / \mathbf{N})} x_{\mathbf{n}},
$$

where $\mathbf{n} / \mathbf{N} \triangleq\left(n_{1} / N_{1}, \ldots, n_{d} / N_{d}\right)$ to be performed elementwise, and the sum denotes the set of nested summations as seen earlier in (1).

Applying the vectorial notation to Equation (2) the inverse of the $d$-dimensional DFT is expressed as follows.

$$
x_{\mathbf{n}}=\frac{1}{\prod_{\ell=1}^{d} N_{\ell}} \sum_{\mathbf{k}=0}^{\mathbf{N}-1} e^{2 \pi i \mathbf{n} \cdot(\mathbf{k} / \mathbf{N})} X_{\mathbf{k}} .
$$

In spite of an apparently more involved formulation, the multidimensional DFT is amenable to a simple interpretation. While the one-dimensional DFT (1D DFT) expresses an input $x_{n}$ as a superposition of sinusoids, the multidimensional DFT expresses its input as a superposition of plane waves, or sinusoids oscillating in space along the directions indicated by $\mathbf{k} / \mathbf{N}$ and having amplitudes defined by $X_{\mathbf{k}}$. Such a decomposition turned out of great importance in practice, for example, in digital image processing $(d=2)$ [11, pp.81-125] or for solving partial differential equations in three or more dimensions $(d \geq 3)$ by the spectral method, i.e., a method by which a linear differential equation is transformed into an ordinary algebraic equation, easily solved. In computational terms the multidimensional DFT can be interpreted as resulting from the composition of a sequence of 1D DFTs along each dimension.

\section{A. A 2-dimensional DFT Spreading System}

In the two-dimensional (2D) case, i.e., where $\mathbf{n}=\left(n_{1}, n_{2}\right)$, from $x_{\mathbf{n}}$ one can first compute $y_{\mathbf{n}^{\prime}}$, which denotes $N_{1}$ independent 1D DFTs of size $N_{2}$ along $n_{2}$ (call them rows) to form a new array, i.e.,

$$
y_{\mathbf{n}^{\prime}}=\sum_{n_{2}=0}^{N_{2}-1} x_{\mathbf{n}} \omega_{2}^{k_{2} n_{2}},
$$

where $\mathbf{n}^{\prime}=\left(n_{1}, k_{2}\right)$, and then compute $X_{\mathbf{k}}$, which denotes $N_{2}$ independent 1D DFTs of size $N_{1}$ along $n_{1}$ (call them columns), to form the final result, i.e.,

$$
X_{\mathbf{k}}=\sum_{n_{1}=0}^{N_{1}-1} y_{\mathbf{n}^{\prime}} \omega_{2}^{k_{1} n_{1}},
$$

where $\mathbf{k}=\left(k_{1}, k_{2}\right)$. Since the nested summations in Equation (1) commute, one can alternately transform first the columns and then the rows. It follows from this commuting property of the multidimensional DFT that, once an efficient way is given to compute a 1D DFT (e.g., an ordinary one-dimensional FFT algorithm), one immediately has a way to efficiently compute the multidimensional DFT. In the two-dimensional case this is known as a row-column algorithm, although there are also intrinsically multidimensional FFT algorithms available in the literature [10], [12]. Obviously, the 2D DFT is just a particular case of the multidimensional DFT and an analogous argument for computing a $d$-dimensional DFT follows unchanged.

At this point we would like to call the reader's attention to the spreading effect of the multidimensional DFT, as indicated for example in (3), in the sense that a given value of $x_{\mathbf{n}}$, which we may call a symbol in the $\mathbf{n}$-domain, is spread by means of the multidimensional DFT along each dimension of the corresponding transform domain, or k-domain. In multidimensional spreading, a dimension can designate time, or frequency, or space, etc. As it is widely known, a diversity scheme is a method for improving the reliability of a signal by utilizing two or more communication channels with different characteristics. This is a very important technique in modern telecommunications to compensate for multipath fading and interference, thus combating burst errors. Diversity is based on the fact that individual channels experience different levels of fading and interference. Therefore, we can obtain more diversity gain if sending one symbol through a multi-dimensional channel because the corresponding diversity gain will increase if the number of dimensions is increased.

As an application of a 2D spreading system, we propose in Section IV a space-frequency system, where an element of the data 2D array spreads into the space and the frequency domains. The space dimensional spreading size is given by the number of antennas. A space-frequency spreading system could be useful for relay communication [19] or sensor network [20] which can have many antennas. In particular, it is more useful for a condition where the signal strengths for each relay (or sensor) antenna are different and where noise and interference in independent wireless channels are different.

\section{Coded 2D Time-Frequency Spreading System}

In this section we introduce a coded 2D time-frequency spreading system. The coded 2D time-frequency spreading transmitter is illustrated in Figure 1, where $a_{i j}$ denotes a symbol at the input to the turbo product code (TPC) encoder [19]. The TPC is based on the product of two component codes which are used in a 2-dimensional matrix form [13]. The $k_{x}$ information bits in the rows are encoded into $n_{x}$ bits using the component $\left(n_{x}, k_{x}\right)$ block code specified for the composite code. After encoding the rows, the columns are encoded using a block code $\left(n_{y}, k_{y}\right)$, where the check bits of the first code are also encoded. The overall block size $n$ of such 
a product code is $n=n_{x} n_{y}$, the total number $k$ of information bits is $k=k_{x} k_{y}$ and the code rate $R$ is $R=R_{x} R_{y}$, where $R_{x}=k_{x} / n_{x}$ and $R_{y}=k_{y} / n_{y}$.

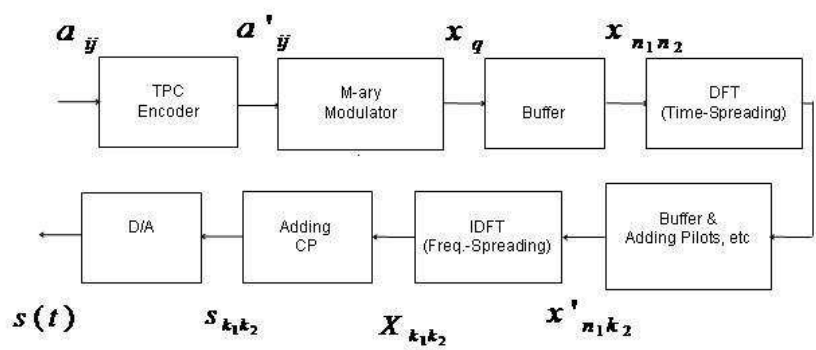

Fig. 1. 2-dimensional (Time-Frequency) spreading transmitter system.

Assuming that $x_{q}$ represents a complex-valued signal point in an $M$-ary constellation, for example, assuming it is $x_{q}=$ $a_{i j}^{\prime}+i a_{i+1, j}^{\prime}$ if QPSK modulation is employed. After modulation, the $x_{q}$ values are fed to a buffer of size $N_{2}$ which stores the incoming $x_{q}$ symbols, forming blocks of $N_{2}$ of them which is the 2D DFT size in the column direction. Therefore, $x_{n_{1} n_{2}}$ enters DFT block and one element of $x_{n_{1} n_{2}}$ spreads to a whole column as follows.

$$
x_{n_{1} k_{2}}^{\prime}=\sum_{n_{2}=0}^{N_{2}-1} \omega_{2}^{k_{2} n_{2}} x_{n_{1} n_{2}},
$$

where $0 \leq k_{2} \leq N_{2}-1$ and $0 \leq n_{1} \leq N-1$.

On the next step, we store the DFT symbol into the buffer in a column by column basis as illustrated in Figure 2 and then the pilots, DC, and guard interval are inserted along a column direction. Therefore, we compute an IDFT of size $N_{1}$ in the row direction, having for input the symbols $x_{n_{1} k_{2}}^{\prime}$, in a row by row basis. The IDFT output, denoted as $X_{k_{1} k_{2}}$, is the time domain OFDM symbol expressed as follows.

$$
X_{k_{1} k_{2}}=\frac{1}{N_{1}} \sum_{n_{1}=0}^{N_{1}-1} \omega_{1}^{-k_{1} n_{1}} x_{n_{1} k_{2}}^{\prime},
$$

where $0 \leq k_{1} \leq N_{1}-1$ and $0 \leq k_{2} \leq N_{2}-1$.

After adding to each row a cyclic prefix containing $N_{g}$ samples we get,

$$
s_{k_{1} k_{2}}=\frac{1}{N_{s}} \sum_{n_{1}=0}^{N_{s}-1} x_{n_{1} k_{2}}^{\prime} \omega_{1}^{-k_{1} n_{1}},
$$

where $N_{s}=N_{1}+N_{g},-N_{g} \leq k_{1} \leq N_{1}-1$ and $0 \leq k_{2} \leq$ $N_{2}-1$. The signal $s_{k_{1} k_{2}}$ enters the digital to analogue (D/A) converter to provide the transmit signal $s(t)$.

A 2D time-frequency spreading receiver is illustrated in Figure 3. After the analogue to digital (A/D) conversion, the cyclic prefix is removed in the received signal $r_{k_{1} k_{2}}$ and then the resulting signal $Y_{k_{1} k_{2}}$ enters the DFT block, the output of which is $y_{n_{1} k_{2}}^{\prime}$ and is expressed as follows.

$$
y_{n_{1} k_{2}}^{\prime}=\sum_{k_{1}=0}^{N_{1}-1} Y_{k_{1} k_{2}} \omega_{1}^{k_{1} n_{1}},
$$

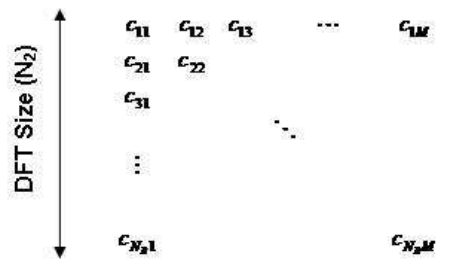

(a)

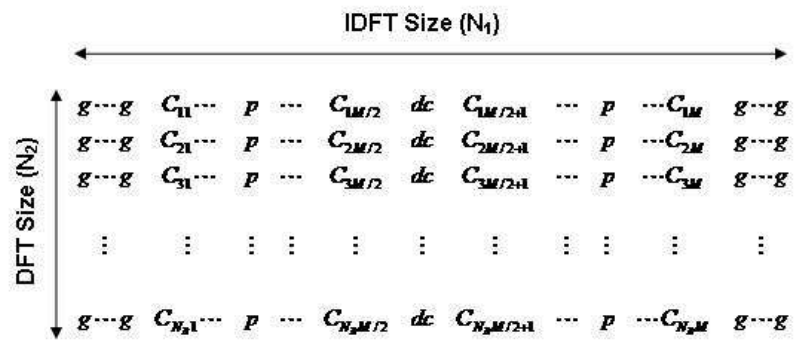

(b)

Fig. 2. First buffer block structure (a) and second buffer block structure in the transmitter.

where $0 \leq n_{1} \leq N_{1}-1$ and $0 \leq k_{2} \leq N_{2}-1$. On the next step, we store $y_{n_{1} k_{2}}^{\prime}$ into the buffer in a row by row manner and then carry out an estimation of the channel frequency response $H_{n_{1} k_{2}}$ based on least squares (LS) estimation (please refer to Appendix [21]) which is given by

$$
H_{n_{1} k_{2}}=\frac{Y_{n_{1} k_{2}}^{p}}{X_{n_{1} k_{2}}^{p}}
$$

where $X_{n_{1} k_{2}}^{p}$ denotes block type pilots we already know and $Y_{n_{1} k_{2}}^{p}$ denotes block type pilots we received. Therefore, we can compensate the OFDM symbol with channel impairment by using $y_{n_{1} k_{2}}^{\prime \prime}$ instead of $y_{n_{1} k_{2}}^{\prime}$ as follows.

$$
y_{n_{1} k_{2}}^{\prime \prime}=H_{n_{1} k_{2}} y_{n_{1} k_{2}}^{\prime} .
$$

After channel estimation, we store $y_{n_{1} k_{2}}^{\prime \prime}$ into the buffer until it is filled with the IDFT of column size $\left(N_{2}\right)$ and we then the symbol in the buffer is output in a column by column manner. Therefore, $y_{n_{1} k_{2}}^{\prime \prime}$ enters the IDFT block to produce $y_{n_{1} n_{2}}$ as follows.

$$
y_{n_{1} n_{2}}=\frac{1}{N_{2}} \sum_{k_{2}=0}^{N_{2}-1} y_{n_{1} k_{2}}^{\prime \prime} \omega_{2}^{-k_{2} n_{2}}
$$

for $0 \leq n_{2} \leq N_{2}-1$ and $0 \leq n_{1} \leq N_{1}-1$.

After obtaining $y_{n_{1} n_{2}}$ with the IDFT, we carry out demodulation, i.e., $y_{n_{1} n_{2}}=b_{i j}^{\prime}+i b_{i+1, j}^{\prime}$ if it is QPSK demodulation. The coded bit $b_{i j}^{\prime}$ is then obtained. The coded bit $b_{i j}^{\prime}$ enters the TPC decoder, and $b_{i j}$ is obtained after the TPC decoding. 


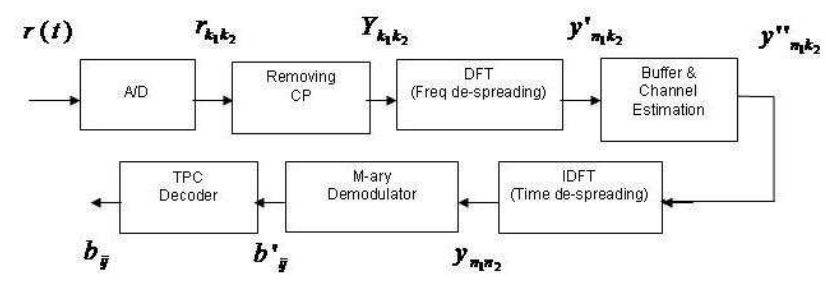

Fig. 3. 2-dimensional spreading (Time-Frequency) receiver system.

\section{A. Spreading Effect}

1) Signal Model: From the receiver process, $y_{n_{1} n_{2}}$ is written as follows.

$$
\begin{aligned}
y_{n_{1} n_{2}} & =\frac{1}{N_{2}} \sum_{k_{2}=0}^{N_{2}-1} y_{n_{1} k_{2}}^{\prime \prime} \omega_{2}^{-k_{2} n_{2}} \\
& =\frac{1}{N_{2}} \sum_{k_{2}=0}^{N_{2}-1} H_{n_{1} k_{2}} y_{n_{1} k_{2}}^{\prime} \omega_{2}^{-k_{2} n_{2}} \\
& =\frac{1}{N_{2}} \sum_{k_{2}=0}^{N_{2}-1} H_{n_{1} k_{2}}\left(\sum_{k_{1}=0}^{N_{1}-1} Y_{k_{1} k_{2}} \omega_{1}^{k_{1} n_{1}}\right) \omega_{2}^{-k_{2} n_{2}} \\
& =\frac{1}{N_{2}} \sum_{k_{2}=0}^{N_{2}-1} \sum_{k_{1}=0}^{N_{1}-1} H_{n_{1} k_{2}} Y_{k_{1} k_{2}} \omega_{1}^{k_{1} n_{1}} \omega_{2}^{-k_{2} n_{2}},
\end{aligned}
$$

for $0 \leq n_{2} \leq N_{2}-1$ and $0 \leq n_{1} \leq N_{1}-1$.

2) Narrowband Interference and Doppler Effect: Narrowband interference and Doppler effect are considered within the transmission bandwidth, assuming that the timing offset is smaller than the guard interval and that no intersymbol interference (ISI) occurs. The received signal, denoted by $r_{n_{1} n_{2}}$, with the cyclic prefix removed and including narrowband interference and frequency offset caused by the Doppler effect is given by

$$
r_{n_{1} n_{2}}=y_{n_{1} n_{2}} e^{2 \pi f_{d}}+I_{k_{1}^{\prime}}+n_{0},
$$

where $f_{d}, I_{k_{1}^{\prime}}$ and $n_{0}$ denote frequency offset, narrowband interference and additive white Gaussian noise (AWGN) sample, respectively. Narrowband interference within transmission bandwidth is expressed as follows.

$$
I_{k_{1}^{\prime}}=\sum_{n_{1}^{\prime}=0}^{N_{n b}-1} i_{n_{1}^{\prime}} e^{\frac{2 \pi i}{N_{n b}} n_{1}^{\prime} k_{1}^{\prime}}, 0 \leq k_{1}^{\prime} \leq N_{n b}-1,
$$

where $N_{n b}$ and $i_{n_{1}^{\prime}}$ denote the number of interfering signals with same subcarrier bandwidth within the transmission bandwidth $N_{1}$ and interference amplitude respectively. Equivalently, the received signal $r_{n_{1} n_{2}}$ is expressed as follows.

$$
\begin{aligned}
r_{n_{1} n_{2}}= & \frac{1}{N_{2}} \sum_{k_{2}=0}^{N_{2}-1} \sum_{k_{1}=0}^{N_{1}-1} H_{n_{1} k_{2}} Y_{k_{1} k_{2}} \omega_{1}^{k_{1} n_{1}} \omega_{2}^{-k_{2} n_{2}} e^{2 \pi f_{d}} \\
& +\sum_{n_{1}^{\prime}=0}^{N_{n b}-1} i_{n_{1}^{\prime}} e^{\frac{2 \pi i}{N_{n b}} n_{1}^{\prime} k_{1}^{\prime}}+N_{0}
\end{aligned}
$$

for $0 \leq n_{2} \leq N_{2}-1,0 \leq n_{1} \leq N_{1}-1$ and $0 \leq k_{1}^{\prime} \leq$ $N_{n b}-1$. It is noticed in Equation (5), that the received signal $Y_{k_{1} k_{2}}$ is spread due to the action of the time domain term $\omega_{1}^{k_{1} n_{1}}$ and the frequency domain term $\omega_{2}^{-k_{2} n_{2}}$. Likewise the effect of narrowband interference and frequency offset is also spread in the frequency domain and in the time domain. In this manner diversity gain is obtained from the time domain and the frequency domain.

\section{A Coded 2D DFT Space-Frequency SpReading SYSTEM}

We propose a coded 2D space-frequency spreading system, having one frequency dimension and one space dimension, based on a MIMO-OFDM system [15]. The coded 2D spacefrequency spreading transmitter is illustrated in Figure 4. It is similar to the coded 2D time-frequency spreading transmitter except that it employs a space-time mapping block. After column spreading, each symbol $z_{n_{1} k_{2}}, 0 \leq k_{2} \leq N_{2}-1$, is mapped into a space-time mapping block $x_{n_{1} k_{2}}^{s_{\ell}}, 0 \leq \ell \leq$ $N_{2}-1$, i.e.,

$$
x_{n_{1} k_{2}}^{s_{\ell}}=M_{\ell}\left(z_{n_{1} k_{2}}\right), \quad 0 \leq \ell \leq N_{2}-1,
$$

where $N_{2}$ denotes the number of antennas, i.e., the spreading size, and $M_{\ell}$ denotes the mapping function. The 2-dimensional space-frequency spreading receiver is illustrated in Figure 5. It is also similar to a 2-dimensional time-frequency spreading receiver except for the space-time de-mapping and combining block. After row de-spreading, each symbol is de-mapped and combined, which we denote as follows.

$$
y_{n_{1} k_{2}}^{s \ell}=D_{\ell}\left(y_{n_{1} k_{2}}^{\prime \prime}\right), \quad 0 \leq \ell \leq N_{2}-1,
$$

where $N_{2}$ denotes the number of antennas and $D_{\ell}$ denotes the de-mapping and combining function.

We consider next a two-input single-output $(2 \times 1)$ channel where a space-time block code [18] is used and $z^{*}$ denotes the complex conjugate of $z$. Each symbol $z_{n_{1} k_{2}}, 0 \leq k_{2} \leq 1$, is mapped into a space-time mapping block $x_{n_{1} k_{2}}^{s_{\ell}}, 0 \leq \ell \leq 1$, as follows.

$$
\begin{aligned}
& {\left[\begin{array}{l}
x_{n_{1} 0}^{s_{0}} \\
x_{n_{1} 1}^{s_{0}}
\end{array}\right]=\left[\begin{array}{c}
z_{n_{1} 0} \\
-z_{n_{1} 1}^{*}
\end{array}\right] \text { for antenna } 1 .} \\
& {\left[\begin{array}{c}
x_{n_{1} 0}^{s_{1}} \\
x_{n_{1} 1}^{s_{1}}
\end{array}\right]=\left[\begin{array}{c}
z_{n_{1} 1} \\
z_{n_{1} 0}^{*}
\end{array}\right] \text { for antenna } 2 .}
\end{aligned}
$$

The data symbols $x_{n_{1} 0}$ and $x_{n_{1} 1}$ spread each to both $z_{n_{1} 0}$ and $z_{n_{1} 1}$. The latter are encoded by space-time coding, and thus spread to each antenna. A received symbol at the output of the channel estimation block in Figure 7, represented by the pair $\left(y_{n_{1} 0}^{\prime \prime}, y_{n_{1} 1}^{\prime \prime}\right)$, can be expressed as follows.

$$
\begin{aligned}
& y_{n_{1} 0}^{\prime \prime}=h_{0} z_{n_{1} 0}+h_{1} z_{n_{1} 1}+n_{0} \\
& y_{n_{1} 1}^{\prime \prime}=-h_{0} z_{n_{1} 1}^{*}+h_{1} z_{n_{1} 0}^{*}+n_{1},
\end{aligned}
$$

where $h_{i}$ and $n_{i}$ denote, respectively, the channel complex multiplicative distortion and a complex random variable representing receiver noise and interference for antenna $i, 0 \leq i \leq$ 1. A received symbol, represented by the pair $\left(y_{n_{1} 0}^{s}, y_{n_{1} 1}^{s}\right)$, after space-time de-mapping can be combined as follows. 
TABLE I

SiMULATION CONFIGURATION FOR 2D CODED TIME-FREQUENCY SPREADING SYSTEM

\begin{tabular}{|c|l|}
\hline Error-Correcting Coding & Turbo product code: \\
& 23 bytes (data block size) \\
& 48 bytes (coded block size) \\
& Component codes $(32,26,4),(16,11,4)$ \\
& Code parameters $\ell_{x}=4, \ell_{y}=2$, \\
$B=8, Q=6$ \\
& Decoder soft quantising: 4 bits \\
& Number of iterations: 3 \\
\hline OFDM parameters & QPSK modulation \\
& DFT size: 256 \\
& Cyclic prefix: $1 / 4$ \\
& Channel bandwidth: $20 \mathrm{MHz}$ \\
\hline Channel & Multipath Rayleigh fading: 4 delay vector \\
& Doppler shift: $50 \mathrm{~Hz}, 70 \mathrm{~Hz}$ \\
& AWGN \\
& Interference: $1.49 \mathrm{MHz}$ within WiMAX \\
& bandwidth \\
\hline Channel estimation & Freq. domain: block-type LS estimation \\
\hline Packet size & $256 \times 64$ (IDFT size $\times$ DFT size \\
& in the transmitter) \\
\hline
\end{tabular}

TABLE II

Simulation CONFIGURATION FOR 2D CODED SPACE-FREQUENCY SPREADING SYSTEM

\begin{tabular}{|c|l|}
\hline $\begin{array}{c}\text { Error-Correcting Coding } \\
\text { and MIMO }\end{array}$ & $\begin{array}{l}\text { Convolutional code of rate 1/2 } \\
\text { Space-time block coding }\end{array}$ \\
\hline OFDM parameters & QPSK modulation \\
& DFT size: 1024 \\
& Cyclic prefix: $1 / 8$ \\
& Channel bandwidth: $10 \mathrm{MHz}$ \\
\hline Channel & MISO $(2 \times 1)$ channel \\
& Multipath Rayleigh fading: 6 delay vector \\
& Doppler shift: $70 \mathrm{~Hz}$ \\
& AWGN \\
& Interference: $1.49 \mathrm{MHz}$ and $2.98 \mathrm{MHz}$ \\
\hline Channel estimation & Freq. domain: block-type LS estimation \\
\hline Packet size & $1024 \times 2$ (IDFT size $\times$ DFT size \\
& in the transmitter) \\
\hline
\end{tabular}

$$
\left[\begin{array}{l}
y_{n_{1} 0}^{s} \\
y_{n_{1} 1}^{s}
\end{array}\right]=\left[\begin{array}{c}
h_{0}^{*} y_{n_{1} 0}^{\prime \prime}+h_{1}\left(y_{n_{1} 1}^{\prime \prime}\right)^{*} \\
h_{1}^{*} y_{n_{1} 0}^{\prime \prime}-h_{0}\left(y_{n_{1} 1}^{\prime \prime}\right)^{*}
\end{array}\right]
$$

Substituting (6) into (7), the pair $\left(y_{n_{1} 0}^{s}, y_{n_{1} 1}^{s}\right)$ can be expressed as follows.

$$
\left[\begin{array}{c}
y_{n_{1} 0}^{s} \\
y_{n_{1} 1}^{s}
\end{array}\right]=\left[\begin{array}{c}
\left(\left|h_{0}\right|^{2}+\left|h_{1}\right|^{2}\right) y_{n_{1} 0}^{\prime \prime}+h_{0}^{*} n_{0}+h_{1} n_{1}^{*} \\
\left(\left|h_{0}\right|^{2}+\left|h_{1}\right|^{2}\right) y_{n_{1} 1}^{\prime \prime}-h_{0} n_{1}^{*}+h_{1}^{*} n_{0}
\end{array}\right] .
$$

\section{Computer Simulation Results}

A computer simulation has been carried out on a $2 \mathrm{D}$ DFT spreading system following WiMAX specifications. It is remarked that if the DFT block in the transmitter is removed as well as the IDFT block in the receiver, the resulting structure is the same as the WiMAX physical layer structure. The main parameters employed in the simulation are given in Table I and in Table II.

Figures 6 and 7 show that the bit error rate performance of the proposed 2D coded spreading system, under Doppler effect and narrowband interference, is better than that of a 1D spreading system (conventional OFDM system) of same size, and presents lower error-floor. For the OFDM in the first simulation, a symbol encoded by a turbo product code and modulated by a QPSK has packet size 256x64. Namely, one symbol is spread to 256 subcarriers in the frequency domain and to 64 time slots in the time domain. In the second simulation, a symbol is also encoded and has packet size $1024 x 2$, i.e., one symbol is spread to 1024 subcarriers in the frequency domain and to two antennas in space domain.

Figure 8 indicates the the proposed 2D coded spreading system has a slightly better performance than a conventional 1D spreading system with a space-time block code. This performance gain difference between a 2D Time-Frequency spreading system and a 2D space-frequency spreading system is caused by the spreading size. The spreading size for a 2D space-frequency spreading system is only 2 because it equals the number of transmitting antennas.

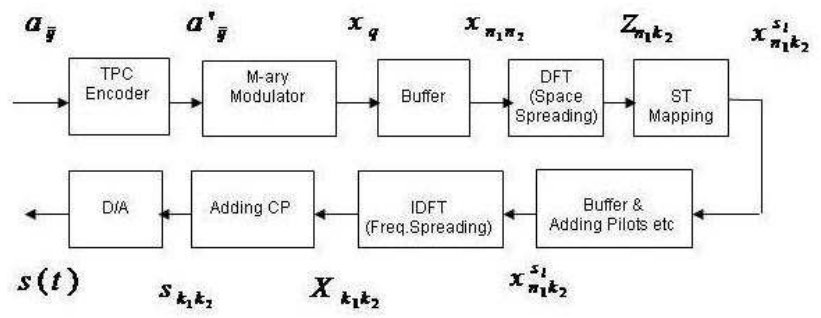

Fig. 4. 2-dimensional space-frequency spreading transmitter system.

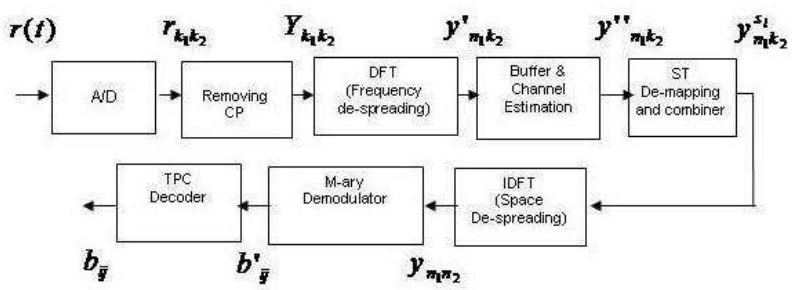

Fig. 5. 2-dimensional space-frequency spreading receiver system.

\section{Closing Remarks}

We have introduced a coded multi-dimensional spreading system using the discrete Fourier transform and shown by example that the $2 \mathrm{D}$ coded spreading system has better performance than the 1D spreading system of the same size, under Doppler effect and narrowband interference. In Section V simulation results of a 2D DFT coded spreading system were presented. We notice that a multi-dimensional DFT spreading system has higher diversity gain than a 1D DFT spreading system. The weak point of the proposed spreading system is that the multi-dimensional spreading system requires a bigger packet size and more DFT blocks are needed so that latency and complexity are increased. The latency and complexity will be increased with increasing spreading size and number of DFT blocks, respectively. If one or more symbols are affected by narrowband interference, deep fading or the Doppler effect, they can still be recovered from the remaining (unaffected) symbols because one symbol is spread to multiple dimensions. 


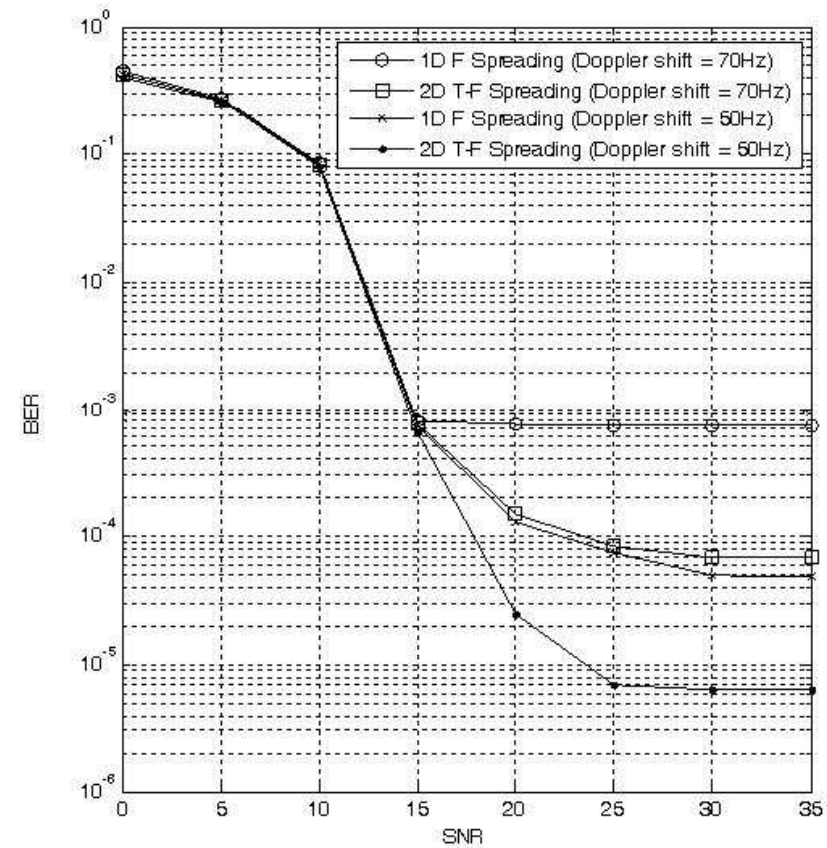

Fig. 6. Comparison of 2-D time-frequency spreading system with 1-D frequency spreading system under the Doppler effect.

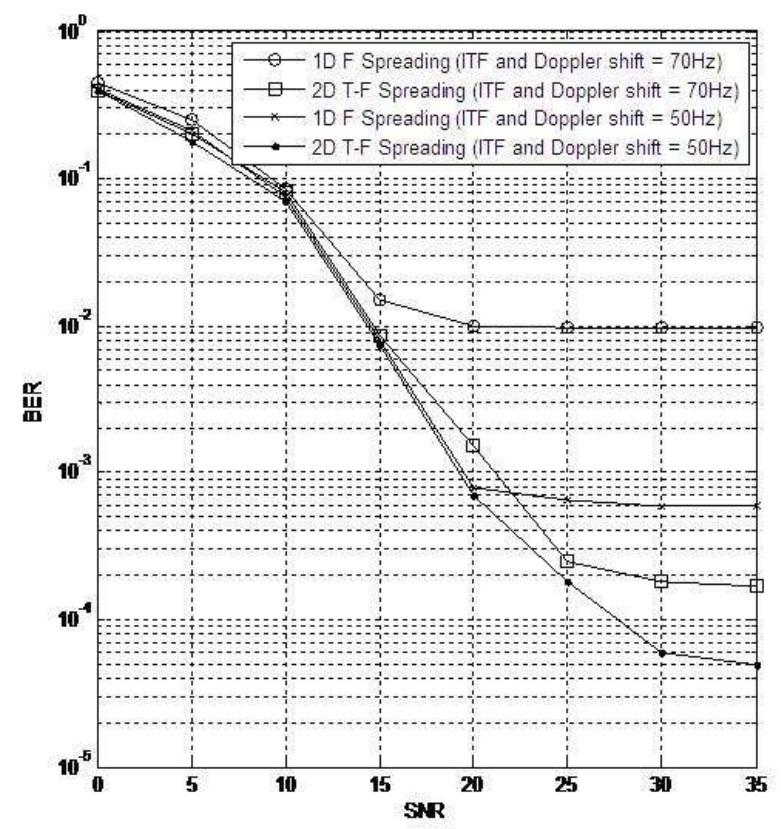

Fig. 7. Comparison of 2-D time-frequency spreading system with 1$\mathrm{D}$ frequency spreading system under the Doppler effect and narrow band interference.

In the case of a 2D time-frequency (or space-frequency) DFT coded spreading system, it spreads not only in the frequency domain but also in the time (or space) domain. In order to compensate for possible error bursts, an interleaver can be used but it is not the same as spreading as meant here.

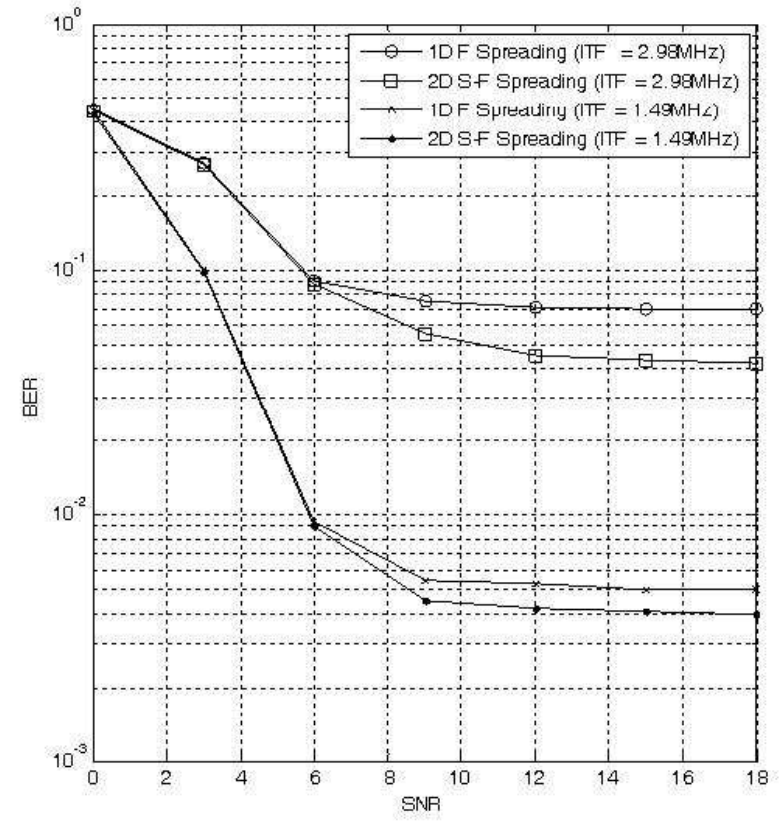

Fig. 8. Comparison of 2-D space-frequency spreading system with 1-D frequency spreading system under narrowband interference.

An interleaver changes error locations but effectively does not reduce the number of errors. Finally, a possible practical application of coded multi-dimensional DFT spreading can be in the form of a coded 2D DFT spreading system, applied to the next generation of WiMAX systems because the 2D DFT spreading architecture is based on the WiMAX architecture.

\section{APPENDIX A}

\section{A Compact Description of the OFDM Technique}

An OFDM system converts a serial data stream into parallel blocks of size $\mathrm{N}$ and modulates these blocks using the inverse fast Fourier transform (IFFT). Time domain samples $x(n)$ of an OFDM symbol are obtained from frequency domain data symbols $X(k)$ as follows.

$$
x(n)=\sum_{k=0}^{N-1} X(k) e^{j 2 \pi n k / N}, 0 \leq n \leq N-1,
$$

where $X(k)$ denotes the data symbol transmitted by the $k$ th subcarrier of the OFDM system, $N$ denotes the fast Fourier transform (FFT) size, and $K$ denotes the set of OFDM subcarriers available for transmission. After the addition of a cyclic prefix $(\mathrm{CP})$ and digital to analogue conversion, the signal is sent through the mobile radio channel. The channel is usually assumed to be constant over an OFDM symbol, but time-varying across OFDM symbols, which is a reasonable assumption for low and medium mobility. At the receiver, the signal contaminated by noise is received. After synchronization, down-sampling, and removal of the $\mathrm{CP}$, the simplified baseband model of the received samples can be represented as follows.

$$
y(n)=\sum_{\ell=0}^{L-1} x(n-l) h(l)+w(n),
$$


where $L$ denotes the number of sample-spaced channel taps, $w(n)$ denotes additive white Gaussian noise (AWGN) samples with zero mean and variance of $\sigma_{w}^{2}$, and the time domain channel impulse response for the current OFDM symbol, $h(\ell)$, is represented as a time-invariant linear filter. In this case, after calculating the FFT of the received signal $y(n)$, the samples in frequency domain can be written as follows.

$$
Y(k)=X(k) H(k)+W(k), \quad k \in K,
$$

where $H$ and $W$ denote the FFTs of $h$ and $w$, respectively.

\section{APPENDIX B \\ Least Squares Channel Estimation}

The least squares (LS) estimate of the channel frequency response $\mathbf{H}$ [21] can be calculated using the received signal and the knowledge of transmitted symbols as

$$
\hat{H}_{L S}=\frac{Y(k)}{X(k)}=H(k)+\frac{W(k)}{X(k)} .
$$

The LS method is the simplest channel estimation method and it is usually used as an initial step for more advanced algorithms.

\section{ACKNOWLEDGEMENT}

Valdemar C. da Rocha Jr. acknowledges partial support of this work by the Brazilian National Council for Scientific and Technological Development - CNPq under grant number 306612/2007-0.

\section{REFERENCES}

[1] ITU-T Recommendation G.992.1 : Asymmetric digital subscriber line (ADSL) transceivers, 07-1999.

[2] IEEE stad 802.16e-2005, 28th Feb 2006

[3] IEEE stad 802.11a-1999 High-speed Physical Layer in the $5 \mathrm{GHz}$ band, 1999-02-11(Retrieved on 2007-09-24).

[4] E. O. Brigham, The Fast Fourier Transform and Its Applications, Prentice-Hall, Englewood Cliffs, NJ, 1988.

[5] A. V. Oppenheim, R. W. Schafer, and J. R. Buck, Discrete-Time Signal Processing, Prentice-Hall, 1999.

[6] R. W. Chang, "Synthesis of band-limited orthogonal signals for multichannel data transmission", Bell System Technical Journal, vol. 46, pp. 1775-1796, 1966

[7] S. B. Weinstein and P. M. Ebert, "Data transmission by frequency division multiplexing using the discrete Fourier Transform", IEEE Trans. Commun. Technol., Vol. COM-19, pp. 628-634, Oct. 1971.

[8] A. J. Viterbi, CDMA : Principles of Spread Spectrum Communication, Prentic Hall PTR, 1995.

[9] H. Kim and G. Markarian, "Turbo product OFDM", Ninth International Symposium on Communication Theory and Applications, 16 - 20 July, 2007, Ambleside, Lake District, UK.

[10] B. Arambepola and P. J. W. Rayner, "Multidimensional fast-Fouriertransform algorithm", Electronics Letters, vol. 15, No. 13, 21st June 1979, pp. 382-383.

[11] R. Gonzales and R. Woods, Digital Image Processing, Addison-Wesley Publishing Company, 1992.

[12] R. Bernardini, "A new multidimensional FFT based on one-dimensional decompositions", IEEE Transactions on Circuits and Systems II: Analog and Digital Signal Processing, Vol. 47, No. 10, Oct 2000, pp.1123 - 1126

[13] G. Drury, G. Markarian and K. Pickavance, Coding and Modulation for Digital Television, Kluwer Academic Publishers, London, 2001.

[14] D. Williams, S. Sonander, N. McSparron, G. Markarian and K. Pickavance, "IEEE 802.16.3 PHY Utilizing Turbo Product Codes", IEEE 802.16 Broadband Wireless Access Working Group, 17th Jan. 2001.
[15] T. C. W. Schenk, P. F.M. Smulders and E. R. Fledderus, "Performance of MIMO OFDM systems in fading channels with additive transmitter and receiver impairments", Proceedings of SPS-DARTS 2005 (the 2005 The first annual IEEE BENELUX/DSP Valley Signal Processing Symposium), pp.41-44.

[16] K. Zhou and Y. H. Chew, "Performance of 2D FFT modulated signal over multipath fading channels", in Proc. 15th IEEE International Symposium on Personal, Indoor, and Mobile Radio Communication, Vol. 2, Sept. 2004, pp. 1337-1341.

[17] T. Riihonen, R. Wichman and T. Roman, "Rate optimization for 2D OFDM relaying", IEEE Communications Letters, Vol.11, No.7, July 2007.

[18] S. M. Alamouti, "A simple transmit diversity technique for wireless communications", IEEE Journal on Select Areas in Communications, Vol. 16, No.8, Oct. 1998.

[19] IEEE802.16j/D4: Part 16: Air interface for Fixed and Mobile Broadband Wireless Access Systems, Multihop Relay Specification. 2008-04-25

[20] K. Römer and F. Mattern, "The design space of wireless sensor networks", IEEE Wireless Communications, Vol.11, No.6, pp.54 61, Dec. 2004

[21] Y. Qiao, S. Yu, P. Su and L. Zhang, "Research on an iterative algorithm of LS channel estimation in MIMO OFDM systems", IEEE Transactions on Broadcasting, Vol.51, No.1, pp. 149-153, March 2005.

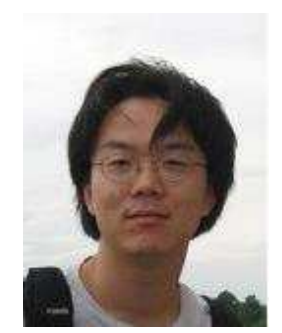

Haesik Kim was born in Seoul, South Korea on 26th September 1975. He received the MS degree from Information and Communications University, South Korea. He worked for Samsung Advanced Institute of Technology as senior research engineer. $\mathrm{He}$ is currently a PhD student in Lancaster University, England. His main research interests are Error Correction Coding, Modulation, BAND AMC, MIMO, OFDM, coopearative communications, etc.

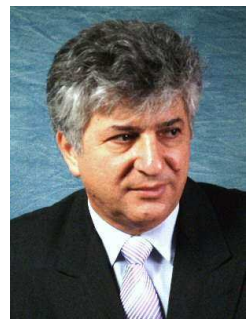

Garik Markarian holds a Chair in Communication Systems and acts as the Head of the Department of Communication Systems of Lancaster University. His reputation as a world's leading scientist was build during his involvement in the IEEE802.16, WIMAX, ETSI BRAN, ETSI HYPERACCESS, 3GPP, DVB-DSNG, DVB-RCS and DVB-S2 standardization bodies, where he chaired a number of working Groups and initiated the development of a number of International Standards. This reputation was cemented by his publications in national and international professional journals (he co-authored over 200 publications, including 4 text books, 39 national and international patents and a great number of papers in international journals), and by his election to a number of national and international Professional Committees. 


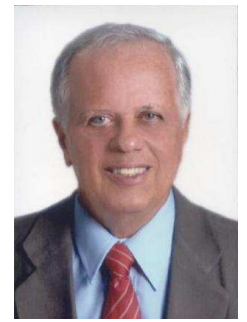

Valdemar C. da Rocha Jr. (M'77, SM'04) was born in Jaboatão, Pernambuco, Brazil, on August 27, 1947. He received in 1970 the B.Sc. degree in Electrical/Electronics Engineering from the Escola Politécnica, Recife, Brazil, and in 1976 he received the Ph.D. degree in Electronics from the University of Kent at Canterbury, U.K. He joined the faculty of the Federal University of Pernambuco, Recife, Brazil, in 1976 as an Associate Professor and founded its Electrical Engineering Postgraduate Programme. He served as Department Chair (19921996), and in 1993 he became Professor of Telecommunications.

$\mathrm{He}$ is the editor for Coding Theory and Techniques, Journal of Communication and Information Systems, co-sponsored by the Brazilian Telecommunications Society and the IEEE Communications Society (http://iecom.dee.ufcg.edu.br/ jcis/), and has been a reviewer for a number of scientific journals including IET Electronics Letters, IET Communications and IEEE Transactions on Information Theory.

He has been involved in the organization of conferences in Brazil and abroad. He was a Co-Chair for the IX Workshop on Signal Processing Applications for Wireless Communications (IEEE SPAWC 2008), Recife, Brazil; Chairman of the Technical Programme Committee, XXV Brazilian Telecommunications Symposium, Recife, Brazil, 2007; Co-Chair for the XV Brazilian Telecommunications Symposium, Recife, Brazil, 1977; Technical Co-Chair of the International Telecommunications Symposium, 2002, Natal, Brazil, and a co-organizer of the Cryptography session of the 1992 IEEE Information Theory Workshop in Salvador, Bahia, Brazil.

During 1990-1992, he was a Guest Professor at the Swiss Federal Institute of Technology-Zurich, Institute for Signal and Information Processing. In 2005-2006 he was a Visiting Professor at the Institute of Integrated Information Systems, University of Leeds, UK, and in 2007 he was a Visiting Professor at the Department of Communication Systems, Lancaster University, UK.

$\mathrm{He}$ is a founder (2002) and past President (2002-2004) of the IEEE Information Theory Society Chapter, Brazil Council. He is founder (2003) and Vice-President for two consecutive terms (2003-2011) of the Institute for Advanced Studies in Communications (IECOM. http://www.iecom.org.br). $\mathrm{He}$ is a founding member (1983) of the Brazilian Telecommunications Society (http://www.sbrt.org.br), served as Vice-President for two terms (2000-2004) and as President also for two terms (2004-2008). He joined the IEEE Communications Society in 1977 and the IEEE Information Theory Society in 1981. He is a member of the Brazilian Society of Applied and Computational Mathematics since 1982, and a Fellow (1992) of the Institute of Mathematics and its Applications, UK.

Prof. da Rocha research interests are in applied digital information theory, including error-correcting codes and cryptography. He has published over 100 engineering and scientific papers, including journal and conference papers, and the book Communication Systems, by Springer, 2005, in co-authorship. 